RESEARCH ARTICLE

\title{
Soil Suitability Assessment for Intercropping Cocoa in Coconut Gardens of Tamil Nadu
}

Sudhalakshmi C

Soil Science and Agricultural Chemistry, Coconut Research Station, Tamil Nadu Agricultural University, Aliyarnagar - 642101.

\begin{abstract}
Geo-referenced soil samples were collected from forty cocoa-growing locations across six districts (Coimbatore, Dindigul, Erode, Namakkal, Salem and Tirunuelveli) of Tamil Nadu. Analysis of data revealed that a vast majority of the samples were alkaline (83\%), non-saline, low in organic carbon (51.3 $\%)$, low in $\mathrm{KMnO}_{4}-\mathrm{N}(75 \%)$, medium in available phosphorus (60\%) and medium in available potassium (51.25\%). The soils were predominantly deficient in DTPA Zn (71.25 \%) and DTPA Cu (32.5\%) in the sampling locations. About $20 \%$ of the cocoa-growing locations were calcareous. The correlation between free $\mathrm{CaCO}_{3}$ content of soil and pod yield revealed that cocoa productivity is drastically affected if the free $\mathrm{CaCO}_{3}$ content of soil is more than $8.0 \%$. Soil suitability assessment is highly imperative before undertaking cocoa intercropping in coconut gardens of Tamil Nadu.
\end{abstract}

Key words: Calcareousness; Cocoa; Coconut; Intercropping; Pod yield; Soil Fertility

\section{INTRODUCTION}

Coconut is an important horticultural crop that exerts a profound influence on the rural economy of the state of Tamil Nadu. It spreads over an area of 4.36 lakh ha with a total production of 5370 million nuts and productivity of 12291 nuts per ha (CDB, 2019). Despite its importance and expanding acreage, the crop turned out to succumb to an array of biotic and abiotic stresses, including rampant pest and disease attack and fluctuating price chart of copra (Maheswarappa et al., 2003). Monocropping of coconut is no longer viable and hence crop intensification is the way forward to transform coconut farming into a viable enterprise to tide over the challenges. Cocoa, nutmeg and banana are widely intercropped with coconut in Tamil Nadu owing to the favorable micro climate. Although cocoa was introduced in India during the early $20^{\text {th }}$ century (Jaganathan et al., 2015), large-scale cultivation started in the late 1980s in Tamil Nadu. The acreage under this crop extends over 29,205 ha in the state (DCCD, 2018), which has been dwindling in the recent past because of an array of cultural and socio-economic factors thus widening the gap between demand and supply of cocoa. The global cocoa demand is on the rise and considering the diminishing income of cocoa farmers, increasing cocoa productivity is highly imperative. Potential cocoa yields are determined based on location and crop-specific characteristics such as climate and crop's natural life cycle (Van Vliet et al., 2015). Soil suitability and soil fertility are highly imperative for improving the productivity of cocoa in the coconut intercropping system.

\section{MATERIAL AND METHODS}

A survey was conducted during 2017 in forty major cocoa growing locations distributed across six districts (Coimbatore, Dindigul, Erode, Namakkal, Salem and Tirunelveli) in Tamil Nadu (Table 1) based on multi-stage stratified sampling method. Details pertinent to the variety of cocoa, age of the plant/ coconut palm, irrigation, nutrient management, plant protection, harvesting, processing, marketing etc., were collected for both coconut and cocoa employing a survey questionnaire. Eighty geo-referenced soil samples were collected from cocoa gardens at 0 - $30 \mathrm{~cm}$ depth. Air-dried samples passed through $2 \mathrm{~mm}$ sieve were utilized for the determination of $\mathrm{pH}$ in 1:2.5 soil water suspension (Jackson, 1973). Available nitrogen $(\mathrm{N})$ was estimated by alkaline permanganametry (Subbiah and Asija, 1956), available phosphorus (P) (Olsen et al., 1954; Bray and Kurtz, 1945), available potassium (K) by Flame Photometry (Stanford and English, 1976), organic carbon (OC) by wet digestion method (Walkley and Black, 1934), free $\mathrm{CaCO}_{3}$ (Dewis and Freita, 1970), DTPA extractable micronutrients by Atomic Absorption Spectrophotometry (Lindsay and Norvell, 1978) and hot water-soluble boron by Azomethin - H reagent method (Berger and Truog, 1939). 
The nutrients were classified as low, medium and high categories for macronutrients and deficient, sufficient for micronutrients based on their soil fertility classification. Nutrient Index Values (NIV) were calculated by employing the procedure of Ramamoorthy and Bajaj, 1969. The index values were rated into various categories viz., low $(<1.67)$, medium (1.67-2.33) and high (>2.33) for OC and available NPK.

\section{(No. of samples in low category $x 1)+$ (No. of samples in medium category $x$ Nutrient Index Value $=\frac{2)+(\text { No. of samples in high category })}{\text { Total number of samples }}$}

\section{RESULTS AND DISCUSSION}

\section{Soil Reaction, Electrical Conductivity and Organic Carbon}

The $\mathrm{pH}$ of the soil samples ranged from 6.09 to 8.54, with a mean value of 7.28 across different sampling locations. About $6.25 \%$ of the samples were acidic in reaction whilst $78.75 \%$ of the samples remained alkaline and only $15 \%$ fell in the neutral range. All the samples were non saline in nature. About 83 \% of soils in Pollachi taluk, Coimbatore district are alkaline and pencil point syndrome is one of the alarming disorders as pointed out by Sudhalakshmi et al (2020). Selvamani and Duraisami (2014) reported that the $\mathrm{pH}$ ranged from 6.6 to 8.8 at $0-30 \mathrm{~cm}$ depth in coconut growing soils of Coimbatore and Tiruppur districts. In the present survey, only localized spots of acidity was witnessed and almost all the horizons were alkaline with kankar nodules.

Organic carbon content was low in $51.3 \%$ of samples and high only in $15 \%$ of samples analyzed. Due to the tropical climate prevailing in Tamil Nadu, there is rapid depletion of soil organic pool, falling in low to medium category. The nutrient index value for organic carbon was in the medium category as per the classification (Tables 1 and 4).

Table 1. Soil Reaction, Electrical Conductivity and Organic Carbon Status of the cocoa-growing soils of Tamil Nadu.

\begin{tabular}{|c|c|c|c|c|c|c|c|c|}
\hline \multirow{2}{*}{$\begin{array}{c}\text { Location } \\
\text { No. }\end{array}$} & \multicolumn{2}{|c|}{ Geographic Co-ordinates } & \multicolumn{2}{|c|}{ pH } & \multicolumn{2}{|c|}{$\begin{array}{c}\text { Electrical } \\
\text { Conductivity }\left(\mathrm{dSm}^{-1}\right)\end{array}$} & \multicolumn{2}{|c|}{ Organic Carbon (\%) } \\
\hline & Location $1\left(L_{1}\right)$ & Location $2\left(\mathrm{~L}_{2}\right)$ & $L_{1}$ & $\mathbf{L}_{2}$ & $L_{1}$ & $L_{2}$ & $L_{1}$ & $\mathbf{L}_{2}$ \\
\hline \multicolumn{9}{|c|}{ Coimbatore District } \\
\hline \multirow{2}{*}{1.} & $10^{\circ} 31.483 \mathrm{~N}$ & $10^{\circ} 31.507 \mathrm{~N}$ & \multirow{2}{*}{7.48} & \multirow{2}{*}{7.46} & \multirow{2}{*}{0.10} & \multirow{2}{*}{0.10} & \multirow{2}{*}{0.54} & \multirow{2}{*}{0.34} \\
\hline & $76^{\circ} 58.160 \mathrm{E}$ & $76^{\circ} 58.147 \mathrm{E}$ & & & & & & \\
\hline \multirow{2}{*}{2.} & $10^{\circ} 29.824 \mathrm{~N}$ & $10^{\circ} 29.825 \mathrm{~N}$ & \multirow{2}{*}{7.43} & \multirow{2}{*}{7.72} & \multirow{2}{*}{0.21} & \multirow{2}{*}{0.16} & \multirow{2}{*}{0.45} & \multirow{2}{*}{0.54} \\
\hline & $76^{\circ} 55.517 \mathrm{E}$ & $76^{\circ} 55.519 \mathrm{E}$ & & & & & & \\
\hline \multirow{2}{*}{3.} & $10^{\circ} 29.483 \mathrm{~N}$ & $10^{\circ} 29.482 \mathrm{~N}$ & \multirow{2}{*}{6.10} & \multirow{2}{*}{6.58} & \multirow{2}{*}{0.22} & \multirow{2}{*}{0.17} & \multirow{2}{*}{0.99} & \multirow{2}{*}{0.57} \\
\hline & $76^{\circ} 54.222 \mathrm{E}$ & $76^{\circ} 54.228 \mathrm{E}$ & & & & & & \\
\hline \multirow{2}{*}{4.} & $10^{\circ} 29.276 \mathrm{~N}$ & $10^{\circ} 29.250 \mathrm{~N}$ & & 609 & 050 & 065 & 128 & 087 \\
\hline & $76^{\circ} 54.205 \mathrm{E}$ & $76^{\circ} 54.194 \mathrm{E}$ & 6.29 & 6.09 & 0.50 & 0.65 & 1.28 & 0.81 \\
\hline 5 & $10^{\circ} 29.251 \mathrm{~N}$ & $10^{\circ} 29.598 \mathrm{~N}$ & 703 & 724 & 10 10 & 05 & 054 & 033 \\
\hline b. & $76^{\circ} 54.194 \mathrm{E}$ & $76^{\circ} 53.400 \mathrm{E}$ & 7.03 & 1.24 & 0.10 & 0.05 & 0.54 & 0.33 \\
\hline 6 & $10^{\circ} 29.594 \mathrm{~N}$ & $10^{\circ} 29.798 \mathrm{~N}$ & 655 & 720 & مחת & م م & 010 & 020 \\
\hline 0. & $76^{\circ} 53.405 \mathrm{E}$ & $76^{\circ} 52.877 \mathrm{E}$ & 0.55 & 1.20 & 0.09 & 0.06 & 0.18 & 0.29 \\
\hline 7 & $10^{\circ} 29.796 \mathrm{~N}$ & $10^{\circ} 32.928 \mathrm{~N}$ & 723 & 721 & 2? ח & 2 0 & 152 & 108 \\
\hline 1. & $76^{\circ} 52.883 \mathrm{E}$ & $76^{\circ} 54.625 \mathrm{E}$ & 1.23 & $1.2 \perp$ & 0.22 & 0.22 & 1.32 & 1.08 \\
\hline 8 & $10^{\circ} 38.008 \mathrm{~N}$ & $10^{\circ} 38.008 \mathrm{~N}$ & 806 & 778 & 00 & 016 & 057 & 036 \\
\hline 8. & $76^{\circ} 53.378 \mathrm{E}$ & $76^{\circ} 53.372 \mathrm{E}$ & 8.06 & 1.18 & 0.09 & 0.16 & 0.51 & 0.36 \\
\hline 9 & $10^{\circ} 37.298 \mathrm{~N}$ & $10^{\circ} 37.298 \mathrm{~N}$ & 735 & 775 & 031 & & & \\
\hline 9. & $76^{\circ} 52.913 \mathrm{E}$ & $76^{\circ} 52.913 \mathrm{E}$ & 1.35 & 1.15 & 0.31 & 0.19 & 0.79 & 1.08 \\
\hline 10 & $10^{\circ} 36.903 \mathrm{~N}$ & $10^{\circ} 36.903 \mathrm{~N}$ & 697 & 704 & 013 & 011 & 099 & 08 \\
\hline 10. & $76^{\circ} 52.651 \mathrm{E}$ & $76^{\circ} 52.651 \mathrm{E}$ & 6.91 & 1.04 & 0.13 & 0.11 & 0.99 & 0.81 \\
\hline 11 & $10^{\circ} 38.108 \mathrm{~N}$ & $10^{\circ} 38.102 \mathrm{~N}$ & 785 & 775 & 023 & & & \\
\hline$\perp \perp$. & $76^{\circ} 53.079 \mathrm{E}$ & $76^{\circ} 53.065 \mathrm{E}$ & 1.85 & 1.15 & 0.23 & 0.22 & 1.08 & 0.70 \\
\hline 12 & $10^{\circ} 37.375 \mathrm{~N}$ & $10^{\circ} 37.375 \mathrm{~N}$ & 771 & 768 & 019 & 0.23 & ח9 0 & 155 \\
\hline & $76^{\circ} 56.192 \mathrm{E}$ & $76^{\circ} 56.192 \mathrm{E}$ & & 1.00 & 0.19 & 0.20 & 0.90 & 1.03 \\
\hline 13 & $10^{\circ} 38.642 \mathrm{~N}$ & $10^{\circ} 38.634 \mathrm{~N}$ & 771 & 788 & 015 & 015 & 0.24 & 036 \\
\hline & $76^{\circ} 57.982 \mathrm{E}$ & $76^{\circ} 57.984 \mathrm{E}$ & & & & & & \\
\hline 14 & $10^{\circ} 39.289 \mathrm{~N}$ & $10^{\circ} 39.298 \mathrm{~N}$ & 671 & 672 & 011 & 011 & 054 & 270 \\
\hline 14. & $76^{\circ} 53.168 \mathrm{E}$ & $76^{\circ} 53.186 \mathrm{E}$ & 0.11 & 0.12 & & & 0.04 & \\
\hline 15 & $10^{\circ} 38.477 \mathrm{~N}$ & $10^{\circ} 38.477 \mathrm{~N}$ & 684 & 700 & 684 & 72 & 20 ח & 36 \\
\hline . & $76^{\circ} 52.910 \mathrm{E}$ & $76^{\circ} 52.910 \mathrm{E}$ & 0.04 & 1.00 & 6.84 & 0.12 & 0.27 & 0.36 \\
\hline
\end{tabular}




\begin{tabular}{|c|c|c|c|c|c|c|c|c|}
\hline 16. & $\begin{array}{l}10^{\circ} 36.495 \mathrm{~N} \\
76^{\circ} 56.216 \mathrm{E}\end{array}$ & $\begin{array}{l}10^{\circ} 36.495 \mathrm{~N} \\
76^{\circ} 56.216 \mathrm{E}\end{array}$ & 7.23 & 7.31 & 0.13 & 0.11 & 0.51 & 0.30 \\
\hline \multicolumn{9}{|c|}{ Dindigul District } \\
\hline \multirow{2}{*}{17.} & $10^{\circ} 24.698 \mathrm{~N}$ & $10^{\circ} 24.698 \mathrm{~N}$ & \multirow{2}{*}{7.91} & \multirow{2}{*}{7.43} & \multirow{2}{*}{0.14} & \multirow{2}{*}{0.24} & \multirow{2}{*}{0.48} & \multirow{2}{*}{0.33} \\
\hline & $77^{\circ} 32.141 \mathrm{E}$ & $77^{\circ} 32.141 \mathrm{E}$ & & & & & & \\
\hline \multirow{2}{*}{18.} & $10^{\circ} 24.634 \mathrm{~N}$ & $10^{\circ} 24.634 \mathrm{~N}$ & \multirow{2}{*}{7.73} & \multirow{2}{*}{7.59} & \multirow{2}{*}{0.14} & \multirow{2}{*}{0.24} & \multirow{2}{*}{0.33} & \multirow{2}{*}{0.24} \\
\hline & $77^{\circ} 32.168 \mathrm{E}$ & $77^{\circ} 32.168 \mathrm{E}$ & & & & & & \\
\hline \multirow{2}{*}{19.} & $10^{\circ} 22.609 \mathrm{~N}$ & $10^{\circ} 22.609 \mathrm{~N}$ & \multirow{2}{*}{7.65} & \multirow{2}{*}{7.70} & 018 & & & \\
\hline & $77^{\circ} 31.252 \mathrm{E}$ & $77^{\circ} 31.252 \mathrm{E}$ & & & 0.18 & 0.19 & 0.36 & 0.27 \\
\hline 20 & $10^{\circ} 23.575 \mathrm{~N}$ & $10^{\circ} 23.575 \mathrm{~N}$ & 774 & & 019 & 026 & 066 & O 31 \\
\hline & $77^{\circ} 32.373 \mathrm{E}$ & $77^{\circ} 32.373 \mathrm{E}$ & 1.14 & 1.62 & 0.19 & 0.26 & 0.66 & 0.31 \\
\hline 21. & $10^{\circ} 24.698 \mathrm{~N}$ & $10^{\circ} 24.698 \mathrm{~N}$ & & & & & & \\
\hline & $77^{\circ} 32.141 \mathrm{E}$ & $77^{\circ} 32.141 \mathrm{E}$ & 7.59 & 7.63 & 0.17 & 0.19 & 0.91 & 0.15 \\
\hline 22 & $10^{\circ} 23.466 \mathrm{~N}$ & $10^{\circ} 23.466 \mathrm{~N}$ & 745 & 790 & 013 & 017 & 013 & 011 \\
\hline 22. & $77^{\circ} 29.177 \mathrm{E}$ & $77^{\circ} 29.177 \mathrm{E}$ & 1.45 & 1.90 & 0.13 & 0.17 & 0.13 & 0.11 \\
\hline 23 & $10^{\circ} 24.249 \mathrm{~N}$ & $10^{\circ} 24.249 \mathrm{~N}$ & 795 & 783 & 012 & 014 & 033 & 20 \\
\hline$\angle 3$. & $77^{\circ} 32.505 \mathrm{E}$ & $77^{\circ} 32.505 \mathrm{E}$ & 1.95 & 1.83 & 0.12 & 0.14 & 0.33 & 0.29 \\
\hline Tirunel & District & & & & & & & \\
\hline 24 & $9^{\circ} 074.930 \mathrm{~N}$ & $9^{\circ} 074.930 \mathrm{~N}$ & 780 & 780 & 013 & 017 & O 31 & 024 \\
\hline 24. & $77^{\circ} 37 \quad E$ & $77^{\circ} 37 \quad E$ & 1.00 & 1.00 & 0.45 & 0.41 & 0.31 & 0.24 \\
\hline 25 & $9^{\circ} 005.280 \mathrm{~N}$ & $9^{\circ} 005.280 \mathrm{~N}$ & 845 & 8.40 & 058 & 050 & 046 & 040 \\
\hline 20. & $77^{\circ} 26528 \mathrm{E}$ & $77^{\circ} 26528 \mathrm{E}$ & 8.45 & 8.40 & 0.58 & 0.50 & 0.46 & 0.40 \\
\hline Erode & & & & & & & & \\
\hline 26 & $11^{\circ} 80.675 \mathrm{~N}$ & $11^{\circ} 18.685 \mathrm{~N}$ & 824 & 821 & 0.47 & 0.48 & 108 & 155 \\
\hline 26. & $77^{\circ} 37.524 \mathrm{E}$ & $77^{\circ} 37.525 \mathrm{E}$ & 8.24 & 8.21 & 0.41 & 0.48 & 1.08 & 1.55 \\
\hline 27. & $11^{\circ} 18.427 \mathrm{~N}$ & $11^{\circ} 18.433 \mathrm{~N}$ & 835 & 816 & 0,50 & & & \\
\hline & $77^{\circ} 37.308 \mathrm{E}$ & $77^{\circ} 37.299 \mathrm{E}$ & 8.35 & 8.16 & 0.50 & 0.40 & 0.49 & 1.41 \\
\hline 28. & $11^{\circ} 15.180 \mathrm{~N}$ & $11^{\circ} 15.175 \mathrm{~N}$ & 8.05 & 8.39 & 0.71 & 0.35 & 1.24 & 0.23 \\
\hline 28 & $77^{\circ} 43.596 \mathrm{E}$ & $77^{\circ} 43.614 \mathrm{E}$ & 8.05 & 8.39 & $0.1 \perp$ & 0.35 & 1.24 & 0.23 \\
\hline 29. & $11^{\circ} 15.325 \mathrm{~N}$ & $11^{\circ} 15.316 \mathrm{~N}$ & 8.19 & 8.04 & 0.25 & 0.45 & 1.13 & 1.10 \\
\hline & $77^{\circ} 43.728 \mathrm{E}$ & $77^{\circ} 43.720 \mathrm{E}$ & 8.19 & 8.04 & 0.25 & & & \\
\hline 30 & $11^{\circ} 15.000 \mathrm{~N}$ & $11^{\circ} 15.005 \mathrm{~N}$ & 780 & 815 & 0.54 & 0.40 & 148 & 1.41 \\
\hline & $77^{\circ} 44.302 \mathrm{E}$ & $77^{\circ} 44.301 \mathrm{E}$ & 1.80 & & & & & \\
\hline Namal & District & & & & & & & \\
\hline 31 & $10^{\circ} 29.291 \mathrm{~N}$ & $11^{\circ} 18.668 \mathrm{~N}$ & 812 & 816 & 031 & 0.45 & 033 & 0.49 \\
\hline $3 \perp$. & $76^{\circ} 58.254 \mathrm{E}$ & $78^{\circ} 17.778 \mathrm{E}$ & 8.12 & 8.16 & & & & \\
\hline 32 & $11^{\circ} 18.640 \mathrm{~N}$ & $11^{\circ} 18.662 \mathrm{~N}$ & 834 & 8.42 & 028 & 036 & 029 & 033 \\
\hline & $78^{\circ} 17.837 \mathrm{E}$ & $78^{\circ} 17.927 \mathrm{E}$ & & & & 0.36 & & \\
\hline 33 & $11^{\circ} 38.631 \mathrm{~N}$ & $11^{\circ} 38.627 \mathrm{~N}$ & 814 & 832 & 014 & 028 & 036 & 0,54 \\
\hline 33. & $78^{\circ} 17.968 \mathrm{E}$ & $78^{\circ} 17.982 \mathrm{E}$ & 8.14 & 8.32 & 0.14 & 0.28 & 0.36 & 0.54 \\
\hline 34 & $11^{\circ} 18.733 \mathrm{~N}$ & $11^{\circ} 18.730 \mathrm{~N}$ & 822 & 786 & 026 & 051 & 026 & 048 \\
\hline 34. & $78^{\circ} 17.542 \mathrm{E}$ & $78^{\circ} 17.540 \mathrm{E}$ & 8.22 & 1.86 & 0.26 & 0.51 & 0.26 & 0.48 \\
\hline 35 & $11^{\circ} 18.680 \mathrm{~N}$ & $11^{\circ} 18.674 \mathrm{~N}$ & 798 & 764 & 054 & 0.4 & 049 & 040 \\
\hline 30. & $78^{\circ} 17.609 \mathrm{E}$ & $78^{\circ} 17.608 \mathrm{E}$ & 1.90 & 1.04 & 0.34 & $0.4 r$ & 0.45 & 0.40 \\
\hline Salem & rict & & & & & & & \\
\hline 36 & $11^{\circ} 45.429 \mathrm{~N}$ & $11^{\circ} 45.420 \mathrm{~N}$ & 8.24 & 832 & 0.34 & 0.27 & 0.52 & 1.73 \\
\hline 30. & $77^{\circ} 48.013 \mathrm{E}$ & $77^{\circ} 48.008 \mathrm{E}$ & 0.24 & & & & & \\
\hline 37. & $11^{\circ} 45.868 \mathrm{~N}$ & $11^{\circ} 45.838 \mathrm{~N}$ & 8.41 & 796 & 0.19 & 0.23 & 096 & 113 \\
\hline & $77^{\circ} 49.547 \mathrm{E}$ & $77^{\circ} 49.507 \mathrm{E}$ & & & & & & \\
\hline 38 & $11^{\circ} 39.812 \mathrm{~N}$ & $11^{\circ} 39.812 \mathrm{~N}$ & 854 & 792 & م20 & 040 & 031 & 063 \\
\hline 38. & $77^{\circ} 47.073 \mathrm{E}$ & $77^{\circ} 47.066 \mathrm{E}$ & 8.54 & 1.92 & 0.20 & 0.49 & 0.31 & 0.03 \\
\hline 39 & $11^{\circ} 36.372 \mathrm{~N}$ & $11^{\circ} 36.370 \mathrm{~N}$ & 818 & 790 & 012 & 0.16 & 0.52 & 0.21 \\
\hline . & $77^{\circ} 47.740 \mathrm{E}$ & $77^{\circ} 44.747 \mathrm{E}$ & & & & & & \\
\hline 40. & $11^{\circ} 31.939 \mathrm{~N}$ & $11^{\circ} 31.945 \mathrm{~N}$ & 8.50 & 8.13 & 0.22 & 0.31 & 1.13 & 1.22 \\
\hline & $77^{\circ} 45.075 \mathrm{E}$ & $77^{\circ} 45.074 \mathrm{E}$ & 8.50 & & & & & \\
\hline
\end{tabular}




\section{Available macronutrients}

The $\mathrm{KMnO}_{4} \mathrm{~N}$ content ranged from 137 to 1140 $\mathrm{kg} \mathrm{ha}^{-1}$ with a mean value of $258 \mathrm{~kg} \mathrm{ha}^{-1}$. Trend analysis revealed that the content was low $(<280 \mathrm{~kg}$ ha $^{-1}$ ) in $75 \%$ of the samples tested, medium in 23.75 $\%$ of the samples and high ( $>450 \mathrm{~kg} \mathrm{ha}^{-1}$ ) in only one sample. Olsen P was low $\left(<11 \mathrm{~kg} \mathrm{ha}^{-1}\right)$ in $10 \%$ of the samples, medium in $60 \%$ of the samples and high (> $22 \mathrm{~kg} \mathrm{ha}^{-1}$ ) in $30 \%$ of the samples analyzed. $1 \mathrm{NNH}_{4} \mathrm{OAc}-\mathrm{K}$ was low $\left(<118 \mathrm{~kg} \mathrm{ha}^{-1}\right)$ in $7.5 \%$ of samples, medium in $51.25 \%$ of samples and high (> $280 \mathrm{~kg} \mathrm{ha}^{-1}$ ) in $41.25 \%$ of samples. About 20 $\%$ of the samples were calcareous and $80 \%$ were non-calcareous. Rao and Batra (1983) established a positive correlation between $\mathrm{pH}$ and ammonia volatilization. In the present study, alkalinity was observed in $78.75 \%$ of soil samples; hence the low status of available nitrogen (60\%) was noticed. The available $\mathrm{P}$ content ranged from 7.0 to 90.0 $\mathrm{kg} \mathrm{ha}^{-1}$ with an average of $22.7 \mathrm{~kg} \mathrm{ha}^{-1}$. About $48 \%$ of the samples fell in the medium category, $30 \%$ in high status and only $10 \%$ of samples were low in available phosphorus. In an earlier survey of coconut growing soils of Coimbatore and Tiruppur districts, Selvamani and Duraisami (2014) reported low status of available phosphorus in 0.76 per cent in coconut growing soils of Coimbatore and Tiruppur districts.

Table 2. $\mathrm{KMnO}_{4}-\mathrm{N}$, Olsen $\mathrm{P}, \mathrm{NNH}_{4} \mathrm{OAc}-\mathrm{K}$ and free $\mathrm{CaCO}_{3}$ of the cocoa-growing soils of Tamil Nadu.

\begin{tabular}{|c|c|c|c|c|c|c|c|c|}
\hline \multirow{2}{*}{$\begin{array}{c}\text { Location } \\
\text { No. }\end{array}$} & \multicolumn{2}{|c|}{$\begin{array}{c}\mathrm{KMnO}_{4}-\mathrm{N} \\
\left(\mathrm{kg} \mathrm{ha}^{-1}\right)\end{array}$} & \multicolumn{2}{|c|}{$\begin{array}{l}\text { Olsen P } \\
\left(\mathrm{kg} \mathrm{ha}^{-1}\right)\end{array}$} & \multicolumn{2}{|c|}{$\begin{array}{c}\text { 1NNH }{ }_{4} \text { OAc - K } \\
\left(\mathrm{kg} \mathrm{ha}^{-1}\right)\end{array}$} & \multicolumn{2}{|c|}{$\begin{array}{c}\text { Free } \mathrm{CaCO}_{3} \\
(\%)\end{array}$} \\
\hline & $\mathbf{L}_{1}$ & $\mathbf{L}_{2}$ & $\mathbf{L}_{1}$ & $\mathbf{L}_{2}$ & $\mathbf{L}_{1}$ & $\mathbf{L}_{2}$ & $\mathbf{L}_{1}$ & $\mathbf{L}_{2}$ \\
\hline \multicolumn{9}{|c|}{ Coimbatore District } \\
\hline 1. & 244 & 244 & 35 & 57 & 108 & 109 & 3.00 & 2.88 \\
\hline 2. & 230 & 213 & 25 & 61 & 186 & 197 & 2.38 & 0.88 \\
\hline 3. & 291 & 314 & 54 & 21 & 408 & 148 & 0.75 & 0.81 \\
\hline 4. & 269 & 1140 & 82 & 90 & 750 & 1092 & 1.38 & 1.31 \\
\hline 5. & 196 & 190 & 29 & 24 & 227 & 132 & 1.75 & 0.31 \\
\hline 6. & 224 & 193 & 63 & 25 & 369 & 113 & 0.25 & 0.44 \\
\hline 7. & 294 & 258 & 31 & 20 & 276 & 328 & 1.19 & 1.56 \\
\hline 8. & 185 & 244 & 61 & 31 & 118 & 160 & 2.31 & 1.63 \\
\hline 9. & 347 & 280 & 28 & 20 & 555 & 299 & 1.56 & 2.38 \\
\hline 10. & 428 & 406 & 24 & 17 & 253 & 290 & 2.69 & 3.19 \\
\hline 11. & 286 & 339 & 20 & 28 & 368 & 360 & 5.38 & 5.88 \\
\hline 12. & 263 & 333 & 16 & 28 & 229 & 364 & 5.63 & 1.31 \\
\hline 13. & 224 & 238 & 12 & 18 & 274 & 188 & 2.00 & 2.69 \\
\hline 14. & 294 & 188 & 14 & 15 & 178 & 180 & 0.25 & 1.88 \\
\hline 15. & 188 & 137 & 15 & 13.2 & 180 & 196 & 1.88 & 0.25 \\
\hline 16. & 260 & 199 & 18.2 & 15 & 100 & 114 & 2.19 & 1.73 \\
\hline \multicolumn{9}{|c|}{ Dindigul District } \\
\hline 17. & 252 & 255 & 20 & 26 & 853 & 266 & 2.88 & 2.25 \\
\hline 18. & 249 & 207 & 15 & 15 & 316 & 336 & 3.19 & 2.00 \\
\hline 19. & 173 & 272 & 20 & 14 & 138 & 151 & 2.56 & 1.31 \\
\hline 20. & 137 & 258 & 17 & 38 & 151 & 170 & 2.69 & 2.44 \\
\hline 21. & 258 & 244 & 15 & 21 & 291 & 298 & 1.88 & 1.88 \\
\hline 22. & 241 & 283 & 14 & 15 & 224 & 255 & 0.25 & 1.00 \\
\hline 23. & 218 & 224 & 16 & 15.2 & 474 & 168 & 0.88 & 1.19 \\
\hline \multicolumn{9}{|c|}{ Tirunelveli District } \\
\hline 24. & 330 & 302 & 20 & 14 & 617 & 241 & 1.81 & 0.44 \\
\hline 25. & 288 & 353 & 17 & 42 & 225 & 637 & 8.20 & 8.75 \\
\hline
\end{tabular}




\begin{tabular}{|c|c|c|c|c|c|c|c|c|}
\hline \multicolumn{9}{|c|}{ Erode District } \\
\hline 26. & 263 & 308 & 15 & 16 & 419 & 445 & 8.75 & 9.00 \\
\hline 27. & 260 & 277 & 21 & 27 & 480 & 573 & 8.20 & 8.75 \\
\hline 28. & 232 & 202 & 16 & 15 & 423 & 405 & 3.31 & 7.64 \\
\hline 29. & 260 & 344 & 15 & 53 & 346 & 911 & 3.44 & 6.85 \\
\hline 30. & 319 & 227 & 26 & 15 & 535 & 320 & 2.88 & 3.94 \\
\hline \multicolumn{9}{|c|}{ Namakkal District } \\
\hline 31. & 198 & 224 & 11 & 16 & 118 & 154 & 3.12 & 1.14 \\
\hline 32. & 137 & 154 & 16 & 13 & 126 & 138 & 2.26 & 2.74 \\
\hline 33. & 156 & 241 & 12 & 18 & 144 & 212 & 1.88 & 1.14 \\
\hline 34. & 188 & 196 & 12 & 10.8 & 242 & 164 & 2.56 & 2.69 \\
\hline 35. & 224 & 218 & 9.8 & 11.1 & 241 & 229 & 2.31 & 1.63 \\
\hline \multicolumn{9}{|c|}{ Salem District } \\
\hline 36. & 241 & 246 & 9.8 & 10.2 & 823 & 791 & 4.25 & 3.25 \\
\hline 37. & 244 & 258 & 12 & 7 & 173 & 239 & 2.19 & 7.81 \\
\hline 38. & 216 & 263 & 9 & 14 & 565 & 738 & 7.94 & 8.00 \\
\hline 39. & 213 & 185 & 9 & 12 & 142 & 120 & 0.94 & 0.63 \\
\hline 40. & 216 & 249 & 11.4 & 10.8 & 110 & 124 & 7.50 & 7.13 \\
\hline
\end{tabular}

The available potassium content ranged from 100 to $1092 \mathrm{~kg} \mathrm{ha}^{-1}$ with an average of $313.9 \mathrm{~kg}$ ha $^{-1}$. About $51.25 \%$ of samples fell in the mediumfertility category $\left(118-280 \mathrm{~kg} \mathrm{ha}^{-1}\right)$ and $41.25 \%$ of the samples were under high fertility status. Motsara (2002) reported that $36 \%$ of the soils of Tamil Nadu fall under the medium category and $52 \%$ under the high status of available potassium. About $20 \%$ of the cocoa soils were calcareous in nature with free $\mathrm{CaCO}_{3}$ content $>5 \%$ and $80 \%$ ranged from $1-5 \%$. Nutrient index values were 1.23 (low) for nitrogen, 2.2 (medium) for phosphorus and 2.33 (medium) for potassium (Tables 2 and 4). Index values of nitrogen, phosphorus and potassium were reported as low, medium and medium for the soils of Salem district of Tamil Nadu (Maragatham et al., 2014).

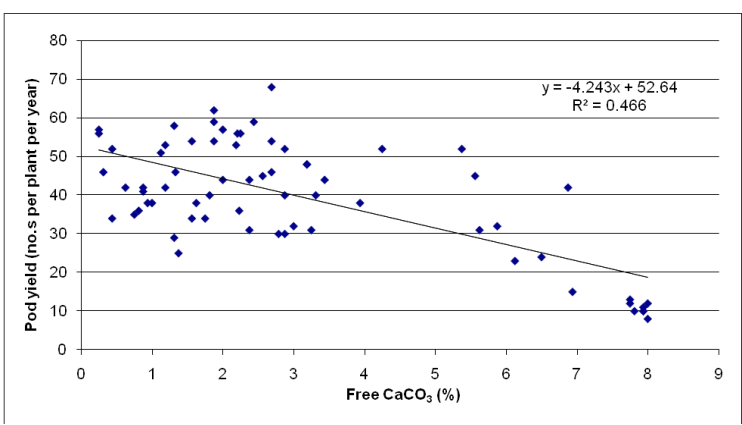

Fig. 1. Correlation between free $\mathrm{CaCO}_{3}$ and pod yield of cocoa

\section{Available micronutrients}

The content of DTPA Fe ranged from 2.40 to $44.6 \mathrm{mg} \mathrm{kg}^{-1}$ with a mean value of $7.28 \mathrm{mg} \mathrm{kg}^{-1}$.
DTPA Fe was sufficient in $83 \%$ of the soil samples and the rest of the samples were deficient. The DTPA Mn content ranged from 1.40 to $17.2 \mathrm{mg} \mathrm{kg}^{-1}$ with a mean value of $7.30 \mathrm{mg} \mathrm{kg}^{-1}$. The nutrient was sufficient (> $2.0 \mathrm{mg} \mathrm{kg}^{-1}$ ) in $92.5 \%$ of soil samples and the deficiency $\left(<2.0 \mathrm{mg} \mathrm{kg}^{-1}\right)$ was witnessed only in $7.5 \%$ of samples. The content of DTPA Zn ranged from 0.13 to $9.45 \mathrm{mg} \mathrm{kg}^{-1}$ with a mean value of $1.50 \mathrm{mg} \mathrm{kg}^{-1}$. DTPA Zn was deficient (<1.2 $\mathrm{mg} \mathrm{kg}^{-1}$ ) in $71.25 \%$ of samples and sufficiency (>1.2 $\mathrm{mg} \mathrm{kg}^{-1}$ ) was noticed across $28.75 \%$ of the samples. The content of DTPA $\mathrm{Cu}$ in cocoa-growing soils ranged from 0.33 to $4.72 \mathrm{mg} \mathrm{kg}^{-1}$ with a mean value of $1.50 \mathrm{mg} \mathrm{kg}^{-1}$. DTPA Cu deficiency $\left(<1.2 \mathrm{mg} \mathrm{kg}^{-1}\right)$ was noticed in $32.5 \%$ of the samples and sufficient (> $1.2 \mathrm{mg} \mathrm{kg}^{-1}$ ) across $67.5 \%$ of the sampling locations. Hot water soluble boron content ranged from 0.75 to $8.52 \mathrm{mg} \mathrm{kg}^{-1}$ with a mean value of 2.24 $\mathrm{mg} \mathrm{kg}^{-1}$ and was sufficient ( $>0.46 \mathrm{mg} \mathrm{kg}^{-1}$ ) in all the sampling locations (Tables 3 and 4).

\section{Correlation between free $\mathrm{CaCO}_{3}$ and pod yield}

A correlation was established between free $\mathrm{CaCO}_{3}$ content of soil and cocoa pod yield (Fig. 1). The pod yield ranged from 8 to 68 pods per plant per year across the cocoa growing locations surveyed. It is highly conspicuous that, of diverse soil fertility parameters, a prominent negative correlation exists between free $\mathrm{CaCO}_{3}$ content of soil and pod yield of cocoa (Fig. 1). As free $\mathrm{CaCO}_{3}$ content of soil increased above $7 \%$, there was a drastic decline in productivity and as it crossed $8.0 \%$, even the very establishment of cocoa plants was difficult. 
Table 3. DTPA Fe, DTPA Mn, DTPA Zn, DTPA Cu and HWS -B of the cocoa-growing soils of Tamil Nadu.

\begin{tabular}{|c|c|c|c|c|c|c|c|c|c|c|}
\hline \multirow{3}{*}{$\begin{array}{c}\text { Location } \\
\text { No. }\end{array}$} & \multicolumn{2}{|c|}{ DTPA Fe } & \multicolumn{2}{|c|}{ DTPA Mn } & \multirow{2}{*}{\multicolumn{2}{|c|}{$\frac{\text { DTPA Zn }}{\left(\mathrm{mg} \mathrm{kg}^{-1}\right)}$}} & \multicolumn{2}{|c|}{ DTPA Cu } & \multicolumn{2}{|c|}{ HWS B } \\
\hline & & & & & & & & & & \\
\hline & $L_{1}$ & $\mathbf{L}_{2}$ & $\mathrm{~L}_{1}$ & $\mathbf{L}_{2}$ & $\mathrm{~L}_{1}$ & $L_{2}$ & $L_{1}$ & $\mathbf{L}_{2}$ & $L_{1}$ & $\mathbf{L}_{2}$ \\
\hline \multicolumn{11}{|c|}{ Coimbatore District } \\
\hline 1. & 6.2 & 7.1 & 6.5 & 9.7 & 0.45 & 0.61 & 1.07 & 2.98 & 3.33 & 2.47 \\
\hline 2. & 9.1 & 7.4 & 10.4 & 9.0 & 0.44 & 0.37 & 1.60 & 1.31 & 1.98 & 2.06 \\
\hline 3. & 28.0 & 22.0 & 13.8 & 12.0 & 0.76 & 0.70 & 1.06 & 0.85 & 8.52 & 3.93 \\
\hline 4. & 27.4 & 44.6 & 7.2 & 7.3 & 3.63 & 5.05 & 1.91 & 1.79 & 2.56 & 3.33 \\
\hline 5. & 13.7 & 5.4 & 17.2 & 6.4 & 2.13 & 0.76 & 0.87 & 0.63 & 1.49 & 1.49 \\
\hline 6. & 15.2 & 13.1 & 10.9 & 9.2 & 1.05 & 1.50 & 1.32 & 1.24 & 2.31 & 1.73 \\
\hline 7. & 9.1 & 11.1 & 11.3 & 12.8 & 3.43 & 1.97 & 1.57 & 1.63 & 3.12 & 1.66 \\
\hline 8. & 5.8 & 4.6 & 8.8 & 4.6 & 0.61 & 1.01 & 0.72 & 0.84 & 1.65 & 2.13 \\
\hline 9. & 7.1 & 5.5 & 2.7 & 2.7 & 1.24 & 2.03 & 1.18 & 1.19 & 2.34 & 1.33 \\
\hline 10. & 8.9 & 5.6 & 4.9 & 7.7 & 1.00 & 0.66 & 1.39 & 1.16 & 2.95 & 1.07 \\
\hline 11. & 10.1 & 6.6 & 7.7 & 11.8 & 2.49 & 4.78 & 3.13 & 4.72 & 6.43 & 1.08 \\
\hline 12. & 6.9 & 5.4 & 9.9 & 13.2 & 1.40 & 1.68 & 1.97 & 1.77 & 4.29 & 6.74 \\
\hline 13. & 4.7 & 11.3 & 11.2 & 8.7 & 1.59 & 1.05 & 1.26 & 1.07 & 3.37 & 4.87 \\
\hline 14. & 2.4 & 4.9 & 2.6 & 10.3 & 0.18 & 0.59 & 0.41 & 1.46 & 0.75 & 0.85 \\
\hline 15. & 4.9 & 6.7 & 10.3 & 9.5 & 0.59 & 0.13 & 1.46 & 0.33 & 0.85 & 1.31 \\
\hline 16. & 7.2 & 5.3 & 6.3 & 8.4 & 1.10 & 0.65 & 1.13 & 1.11 & 2.40 & 1.54 \\
\hline \multicolumn{11}{|c|}{ Dindigul District } \\
\hline 17. & 4.8 & 5.9 & 10.5 & 9.3 & 1.53 & 0.71 & 1.34 & 1.46 & 4.34 & 1.91 \\
\hline 18. & 4.8 & 6.4 & 7.9 & 6.2 & 0.56 & 0.41 & 1.38 & 1.41 & 2.40 & 2.29 \\
\hline 19. & 5.9 & 5.5 & 10.3 & 9.4 & 1.49 & 2.01 & 2.19 & 1.96 & 1.89 & 1.81 \\
\hline 20. & 7.2 & 6.8 & 7.8 & 6.8 & 0.85 & 0.58 & 2.08 & 1.79 & 1.65 & 1.00 \\
\hline 21. & 6.9 & 8.0 & 9.2 & 6.7 & 0.50 & 0.87 & 1.49 & 1.63 & 2.41 & 1.31 \\
\hline 22. & 5.3 & 3.8 & 8.8 & 8.2 & 0.42 & 0.47 & 1.52 & 1.37 & 1.24 & 1.16 \\
\hline 23. & 3.4 & 3.8 & 5.5 & 7.8 & 0.31 & 0.35 & 0.87 & 0.82 & 1.00 & 1.24 \\
\hline \multicolumn{11}{|c|}{ Tirunelveli District } \\
\hline 24. & 3.9 & 4.6 & 14.5 & 10.6 & 1.57 & 0.47 & 1.69 & 1.69 & 2.17 & 2.01 \\
\hline 25. & 6.0 & 7.0 & 7.7 & 4.2 & 0.26 & 0.94 & 1.12 & 1.04 & 2.41 & 3.14 \\
\hline \multicolumn{11}{|l|}{ Erode District } \\
\hline 26. & 4.7 & 4.5 & 9.3 & 6.8 & 1.31 & 0.92 & 1.38 & 1.57 & 2.16 & 1.31 \\
\hline 27. & 3.9 & 4.4 & 6.4 & 9.1 & 1.21 & 1.13 & 1.37 & 1.34 & 1.39 & 1.31 \\
\hline 28. & 4.2 & 4.5 & 6.7 & 6.7 & 1.00 & 0.79 & 1.68 & 1.80 & 1.62 & 1.77 \\
\hline 29. & 3.6 & 7.5 & 6.4 & 6.3 & 0.79 & 1.17 & 1.06 & 1.54 & 2.33 & 3.21 \\
\hline 30. & 6.1 & 4.5 & 11.6 & 7.6 & 9.45 & 8.73 & 1.76 & 1.66 & 4.22 & 1.62 \\
\hline \multicolumn{11}{|c|}{ Namakkal District } \\
\hline 31. & 6.0 & 5.8 & 6.7 & 5.5 & 0.74 & 0.56 & 1.64 & 1.86 & 1.16 & 1.67 \\
\hline 32. & 8.2 & 7.8 & 6.6 & 6.8 & 0.78 & 0.83 & 1.87 & 1.98 & 1.31 & 1.94 \\
\hline 33. & 6.4 & 6.2 & 3.2 & 2.3 & 0.80 & 0.94 & 2.32 & 1.88 & 2.01 & 2.16 \\
\hline 34. & 3.6 & 4.2 & 2.1 & 2.5 & 0.68 & 0.72 & 1.52 & 2.08 & 1.24 & 1.16 \\
\hline 35. & 4.2 & 4.8 & 7.7 & 5.4 & 1.14 & 1.26 & 1.97 & 1.77 & 1.98 & 1.33 \\
\hline \multicolumn{11}{|c|}{ Salem District } \\
\hline 36. & 3.6 & 4.4 & 2.1 & 2.5 & 1.87 & 2.55 & 1.81 & 2.59 & 2.01 & 2.33 \\
\hline 37. & 3.9 & 4.8 & 2.3 & 2.2 & 0.62 & 0.44 & 0.91 & 1.48 & 2.72 & 2.16 \\
\hline 38. & 3.9 & 5.0 & 2.1 & 1.9 & 0.24 & 0.28 & 0.77 & 0.76 & 3.76 & 1.94 \\
\hline 39. & 4.2 & 4.9 & 1.8 & 2.1 & 0.81 & 0.90 & 1.26 & 0.92 & 1.24 & 1.39 \\
\hline 40. & 3.8 & 5.1 & 1.4 & 1.5 & 0.72 & 1.82 & 0.98 & 1.38 & 1.93 & 1.16 \\
\hline
\end{tabular}

$107|10-12| 6$ 
A plethora of evidence states that carbonate activity in calcareous soils influences the rate of volatilization of ammonia (Ryan et al., 1981) and that carbonate affects the rhizospheric processes, especially in acidification factor. The presence of $\mathrm{CaCO}_{3}$ directly or indirectly affects the chemistry and availability of nitrogen, phosphorus, iron, zinc, magnesium, calcium, potassium and copper (Marschner, 1995) through ammonia volatilization and phosphorus precipitation. In addition, iron, zinc and magnesium deficiencies are common in soils with high $\mathrm{CaCO}_{3}$ and alkaline $\mathrm{pH}$ values (Marschner,

Table 4. Nutrient Index Values of macro nutrients in the cocoa-growing soils of Tamil Nadu

\begin{tabular}{|c|c|c|c|c|c|c|}
\hline S. No. & Parameter & $\begin{array}{l}\text { Minimum } \\
\text { value }\end{array}$ & $\begin{array}{c}\text { Maximum } \\
\text { value }\end{array}$ & Mean value & $\begin{array}{l}\text { Standard } \\
\text { Deviation }\end{array}$ & $\begin{array}{c}\text { Nutrient } \\
\text { Index }\end{array}$ \\
\hline 1. & $\mathrm{pH}$ & 6.09 & 8.54 & 7.70 & 0.57 & - \\
\hline 2. & $\mathrm{EC}\left(\mathrm{dSm}^{-1}\right)$ & 0.05 & 6.84 & 0.352 & 0.751 & - \\
\hline 3. & Organic carbon (\%) & 0.11 & 1.73 & 0.65 & 0.42 & 1.88 \\
\hline 4. & $\mathrm{KMnO}_{4} \mathrm{~N}\left(\mathrm{~kg} \mathrm{ha}^{-1}\right)$ & 137 & 1140 & 258 & 115 & 1.23 \\
\hline 5. & Olsen $\mathrm{P}\left(\mathrm{kg} \mathrm{ha}^{-1}\right)$ & 7.0 & 90.0 & 22.7 & 16.3 & 2.20 \\
\hline 6. & $1 \mathrm{NNH}_{4} \mathrm{OAc} \mathrm{K}\left(\mathrm{kg} \mathrm{ha}^{-1}\right)$ & 100 & 1092 & 313.9 & 215.5 & 2.33 \\
\hline 7. & Free $\mathrm{CaCO}_{3}(\%)$ & 0.25 & 9.00 & 3.04 & 2.50 & - \\
\hline 8. & DTPA Fe (mg kg-1 $)$ & 2.40 & 44.6 & 7.28 & 6.15 & - \\
\hline 9. & DTPA Mn $\left(\mathrm{mg} \mathrm{kg}^{-1}\right)$ & 1.40 & 17.2 & 7.30 & 3.48 & - \\
\hline 10. & DTPA Zn (mg kg-1) & 0.13 & 9.45 & 1.31 & 1.55 & - \\
\hline 11. & DTPA Cu (mg kg-1) & 0.33 & 4.72 & 1.50 & 0.62 & - \\
\hline 12. & Hot water soluble $B\left(\mathrm{mg} \mathrm{kg}^{-1}\right)$ & 0.75 & 8.52 & 2.24 & 1.34 & - \\
\hline
\end{tabular}

1995). Hillel (1986) recorded that emergence of bean seedlings in fine sandy loam soil was reduced from 100 to $0 \%$ when crust strength increased from 108 to 273 mbar because of calcareousness. The growers of cocoa commonly noticed Lime- induced iron chlorosis in calcareous soils. On foliar spraying with micronutrients, the plants reverted back to normalcy only for a brief period of time and again they turned chlorotic. In intensely calcareous soils, there was problem even with the very establishment of cocoa seedlings and it was more conspicuous in Erode and Salem districts of Tamil Nadu. Thus it can be concluded that cocoa cultivation is not remunerative if free $\mathrm{CaCO}_{3}$ content in the soil is > $8 \%$.

\section{CONCLUSION}

From the survey conducted across six prominent cocoa growing districts of Tamil Nadu, it was found that about $78.75 \%$ of the soils were alkaline in reaction and the electrical conductivity was nonsaline across the sampling locations. The low status of $\mathrm{KMnO}_{4}-\mathrm{N}$, Olsen $\mathrm{P}$ and $1 \mathrm{NNH}_{4} \mathrm{OAc}-\mathrm{K}$ was witnessed in $75 \%, 10 \%$ and $7.5 \%$ of the sampling locations respectively. About $20 \%$ of the cocoagrowing locations surveyed were calcareous in nature with free $\mathrm{CaCO}_{3}$ content $>5 \%$. DTPA Fe was deficient across $17 \%$, DTPA Mn across $7.5 \%$, DTPA Zn over $71.2 \%$ and DTPA Cu in $32.5 \%$ of the sampling locations. Hot water soluble boron was sufficient all through the sampling locations. The correlation between free $\mathrm{CaCO}_{3}$ and pod yield was negative. Pod yield is drastically affected to the tune of $<10$ pods per plant per year in intensely calcareous soils. Thus the survey revealed that intercropping of cocoa is not remunerative if free $\mathrm{CaCO}_{3}$ content in the soil is $>8.0 \%$. Soil testing and crop suitability assessment are imperative for undertaking intercropping of cocoa in coconut gardens of Tamil Nadu to avoid huge economic losses by cultivating in unsuitable soils.

\section{REFERENCES}

Berger, K.C. and E.Troug. 1939. Boron determination in soils and plants using the quinalizarin reaction. Indus. \& Engg. Chem., Analytical Edition. 11: 540-545.

Bray, R.H. and L.T. Kurtz. 1945. Determination of total, organic and available forms of phosphorus in soils. Soil Sci., 59: 39-45.

Coconut Development Board Statistics. 2019. https:// coconutboard.gov.in/Statistics.aspx

Dewis, J. and F. Freitas. 1970. Calcium carbonate content - Acid neutralization in physical and chemical methods of soil and water analysis. Food and Agricultural Organization of the United Nations, Rome, Italy, III 2-2, P.71-72.

Directorate of Cashew and Cocoa Development. 2018. Technical Bulletin No. 134. Cocoa Guide. 1-52.

Hillel, P., 1986. Biochemistry of Fern Spore Germination: Protease Activity in Ostrich Fern Spores. PI. Physiol., 80: 992-996.

Jackson, M.L. 1973. Soil Chemical Analysis. Prentice Hall of India, New Delhi, p.498. 
Jaganathan, C., Thamban, C.T., Jose, S., Jayasekhar,S. Muralidharan, K. and K.P.Chandran. 2015. Analysis of organic farming practices in cocoa in India. J. Plant. Crops., 43 (2): 131- 138.

Lindsay, W.L. and W.A.Norvell. 1978. Development of a DTPA soil test for zinc, iron, manganese and copper. Soil Sci. Soc. Am. J., 42: 421-428.

Maragatham, S., Santhi,R., Radhika,K., Sivagnanam,S., Rajeswari, R., Hemalatha,S., Kanimozhi, A., Pradip Dey and A.Subba Rao. 2014. An appraisal of available nutrients status and soil fertility mapping for Salem district of Tamil Nadu. Madras Agric. J., 101 (1-3): 51-58.

Marschner, H., 1995. Mineral Nutrition of Higher Plants. Academic Press, London.

Motsara, M.R. 2002, Available NPK Status of Indian Soils as depicted by soil fertility maps. Fert. News., 47 (8): 15-21.

Ramamoorthy, B. and Bajaj, J.C. 1969. Available N, $\mathrm{P}$ and $\mathrm{K}$ status of Indian soils. Fert. News., 14: 24-26.
Rao, D.L.N. and Lalita Batra. 1983. Ammonia volatilization from applied nitrogen in alkali soils. PI. and Soil., 70: 219-228.

Ryan, J., Ayubi, A.G. 1981. Phosphorus availability indices in calcareous Lebanese soils. PI. and Soil., 62: 141-145. https://doi.org/10.1007/ BF02205034.

Sudhalakshmi, C., Sindhu, T., Rahamath Nisha,J., Venkatesan, K. and V.Ramaswami. 2020. Survey of pencil point disorder manifested coconut gardens of Pollachi taluk, Coimbatore Dt, Tamil Nadu. Int. J. of Chem. Studies., 8(6): 97-101.

Van Vliet, Jiska, A., Slingerland, Maja Giller and E.Ken. 2015. Mineral nutrition of cocoa: A review. 57 pp. Wageningen University and Research Centre, Wageningen. ISBN: 978-94-6257-705-3

Walkely and C.A.Black. 1934. An examination of digestion method for determining organic carbon in soil, effect of variation in digestion conditions and of inorganic soil constituents. Soil Sci., 63: 251-263. 